\title{
On transferring new constant pressure heat capacity computation methods to engineering practice
}

\author{
S. Rajaeirad ${ }^{1}$, N. Dadgostar ${ }^{1}$, H. Loria ${ }^{2}$, M. Satyro ${ }^{2}$, and J.M. Shaw ${ }^{1}$ \\ ${ }^{1}$ Department of Chemical and Materials Engineering, University of Alberta, Edmonton, Canada \\ ${ }^{2}$ Virtual Materials Group, Calgary, Canada
}

Pressure-volume-temperature relationships (equations of state) and energy models are two pillars underlying calculations in the chemical process industries. While, equations of state tend to be the focus of research, development and customization, energy models appear to be underdeveloped even though accurate and generalized energy models are needed for sizing individual pieces of heat transfer equipment (i.e.: heaters/furnaces, heat exchangers, chillers/coolers), and for process integration tasks. Thus energy models also impose implicit and explicit capital and operating cost consequences for processes designed using them. These uncertainties and their consequences are frequently invisible to users of simulation software and even more so as processes designed around ill-defined fluids, or fluids where practitioners have limited experience (e.g.: heavy oils, bio-fuels) become more important, or where the proposed operating conditions are outside common practice.

There are two general approaches for computing constant pressure heat capacities for fluids. One is based on generalized correlations for ideal gases plus departure functions based on equations of state to address non-ideal gases and liquids. The other is to compute liquid phase heat capacities directly from generalized correlations for liquids, which can be used independently, or in combination with departure functions to back calculate non-ideal gas or ideal gas heat capacities, or to validate liquid heat capacities computed from ideal gas + departure function based calculations. Generalized ideal gas and liquid correlations, based on the concept that compounds or mixtures possessing the same number of atoms per unit mass possess the same specific heat capacity were recently developed [1, 2]. The concept is illustrated in Figure 1 for liquids where molecular structure, molar mass, and the nature of the elements present have only secondary impacts on heat capacity if the numbers of atoms per unit mass are the same. The ideal gas correlation is shown to be preferred over four Lee-Kesler correlations and the Harrison-Seaton correlation on the basis of accuracy and range of application for general-purpose calculations. For large molecules, the correlation approaches the accuracy of the benchmark Benson method, even though no compound specific information other than elemental composition is required. The correlation for liquids also provides accurate heat capacity values for a broad range of fluids at saturation and is, for example, preferred over the widely used LeeKesler correlation for liquids based both on accuracy and range of application.

Both the liquid and ideal gas correlations are simple and predictive, and well suited for inclusion in process simulators. Implementation was expected to be straightforward. For compounds, or mixtures comprising constituents defined on a molecular basis, elemental compositions of streams are readily calculated. For mixtures defined on other bases, American Petroleum Institute standards can be applied to obtain elemental composition, or elemental analysis can be measured exogenously and included in the input data set. In practice, significant sources for dissonance have arisen: between computed and measured elemental analysis, and between computed differences between liquid and ideal gas heat capacities based on correlations and departure functions. While these correlations have been made available as options in a process simulator [3], these sources of dissonance have complicated the implementation of the heat capacity correlations and underscore the underdeveloped nature of energy modeling in process calculations more broadly. 

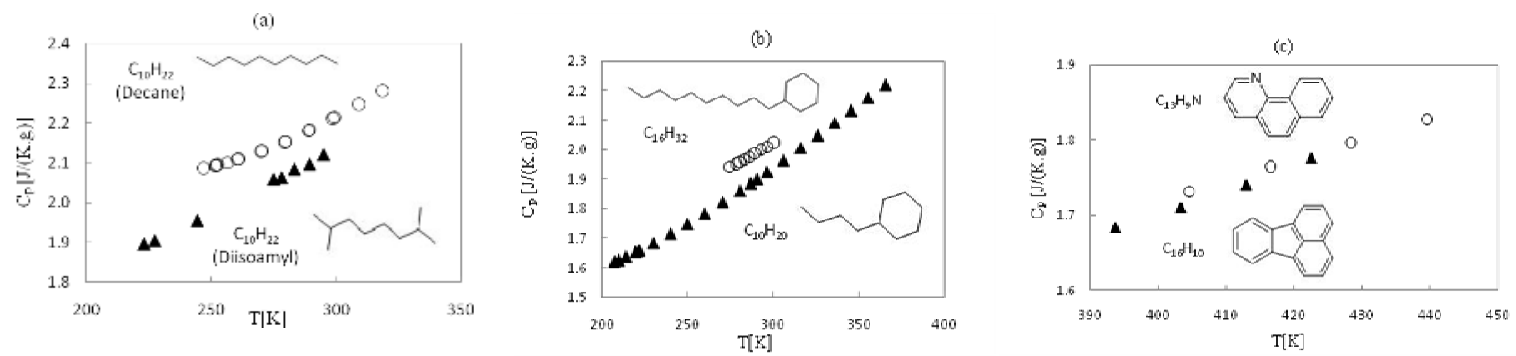

Figure 1: Experimental specific liquid heat capacity values for: (a) $\boldsymbol{\Delta}, 2,7$-Dimethyloctane, Diisoamyl $\left(\mathrm{C}_{10} \mathrm{H}_{22}\right)$ and $\circ$, Decane $\left(\mathrm{C}_{10} \mathrm{H}_{22}\right)$; (b) $\boldsymbol{\Delta}$, Butylcyclohexane $\left(\mathrm{C}_{10} \mathrm{H}_{20}\right)$ and $\circ$, Decylcyclohexane $\left(\mathrm{C}_{16} \mathrm{H}_{32}\right)$; (c) $\boldsymbol{\Delta}$, Fluoranthene $\left(\mathrm{C}_{16} \mathrm{H}_{10}\right)$ and $\circ$, Benzo[h]quinoline $\left(\mathrm{C}_{13} \mathrm{H}_{9} \mathrm{~N}\right)$.

\section{References}

1. N. Dadgostar, J.M. Shaw, Fluid Phase Equilibria 31325 (2012)

2. V. Lastovka, J.M. Shaw, Predictive correlations for ideal gas heat capacities of pure hydrocarbons and petroleum fractions (in preparation)

3. VMGSIM ${ }^{\mathrm{TM}}$ 7.0, Virtual Materials Group, October 4, 2012 